\title{
基于萗基新型冠醚的合成、晶体结构及其对联吡啶盐的络合性能研究
}

\author{
宗乾收*, $a, b, c$ 吴建一 $a, b$ \\ ( ${ }^{a}$ 嘉兴学院生物与化学工程学院 嘉兴 314001) \\ ${ }^{b}$ 嘉兴市化工清洁工艺重点实验室, 浙江嘉兴 314001) \\ ( ${ }^{c}$ 浙江大学药学院 杭州 310058)
}

\begin{abstract}
摘要 以 2,7-二羟基萗为起始原料, 经与 8-(对甲苯磺酰氧基)-3,6-二氧-1-辛醇醚化，再与对甲苯磺酰氯酯化得 7, 7 最后 与 2,7 -二羟基萘在碳酸铯为碱作用下得基于 2,7 -二羟基荎基块新型的冠醚, 三步总收率为 $35 \%$. 利用单晶 $X$ 衍射研究其 结构, 晶体为三斜晶系, $P-1$ 空间群, $a=8.165(3) \AA, b=13.435(4) \AA, c=14.083(3) \AA, \gamma=64.11(2)^{\circ}, \beta=80.40(3)^{\circ}, \gamma=$ $88.19(3)^{\circ}, V=1368.9(7) \AA^{3}, Z=2, D_{\mathrm{c}}=1.331 \mathrm{~g} / \mathrm{cm}^{3}, \lambda=0.071070 \mathrm{~nm}, \mu(\mathrm{Mo} \mathrm{K \alpha})=1.331 \mathrm{~mm}^{-1}, M_{\mathrm{r}}=353.22, F(000)=584$. 在 1 的晶体结构中, 两个菜基块通过二缩三乙二醇醚键相连形成 1 个二萗并[30]-冠- 8 大环结构, 空腔大小为 $8.2 \AA \times$ $12.0 \AA$, 分子呈 $Z$ 型结构. 通过核磁研究了主体分子 1 对联吡啶盐在溶液中的相互络合作用.
\end{abstract}

关键词 冠醚; 合成; 晶体结构; 络合; 联吡啶盐

\section{Novel Naphthalene-Based Crown Ether: Synthsis, Crystal Structure and Its Complexation with Paraquat Derivatives}

\author{
Zong, Qianshou* ${ }^{*, a, b}$ \\ Wu, Jianyi ${ }^{a, b}$ \\ ( ${ }^{a}$ Department of Biology and Chemical Engineering, Jiaxing University, Jiaxing 314001) \\ ( ${ }^{b}$ Key Laboratory of Chemical Cleaning Technology, Jiaxing City, Jiaxing 314001) \\ ( ${ }^{c}$ School of Pharmaceutical Sciences, Zhejiang University, Hangzhou 310058)
}

\begin{abstract}
A Novel naphthalene-based crown ether has been synthesized by the etherification of 2,7-dihydroxynaphthalene with 8-tosyloxy-3,6-dioxaoctanol in the presence of $\mathrm{K}_{2} \mathrm{CO}_{3}$, followed by reaction with $p$-toluenesufonyl chloride to give 7 . Compound 7 was further reacted with 2,7-dihydroxynaphthalene in the presence of cesium carbonate to give $\mathbf{1}$ in $38 \%$ total yield. Its crystal structure was determined by X-ray diffraction method. The crystal is of triclinic, space group P-1 with $a=$ 8.165(3) $\AA, b=13.435(4) \AA, c=14.083(3) \AA, \alpha=64.11(2)^{\circ}, \beta=80.40(3)^{\circ}, \gamma=88.19(3)^{\circ}, V=1368.9(7) \AA^{3}, Z=2, D_{\mathrm{c}}=1.331$ $\mathrm{g} / \mathrm{cm}^{3}, \lambda=0.071070 \mathrm{~nm}, \mu(\mathrm{Mo} \mathrm{K} \alpha)=1.331 \mathrm{~mm}^{-1}, M_{\mathrm{r}}=353.22, F(000)=584$. The crystal structure of 1 shows that two napthalene units are linked two tri(ethylene glycol) bridges to form dinapthalzo[30]crown- 8 cavity and one $Z$-like central cavity with size of $c a .8 .2 \AA \times 12.0 \AA$. The host 4 was proved to be a efficient host for the complexation with paraquat derivatives in solution by ${ }^{1} \mathrm{H}$ NMR.
\end{abstract}

Keywords crown ethers; synthesis; crystal structure; complexation; paraquat derivatives

自 1967 年 Pedersen ${ }^{[1]}$ 首次发现冠醚以后，冠醚的特 殊性质立刻引起各国化学家的重视, 大量类似结构的冠 状化合物被合成出来. 冠醚作为超分子化学第一代的主 体分子, 经过四十多年的研究在新型冠醚的合成、识别 及组装研究方面已取得了丰硕的研究成果 ${ }^{[2 \sim 8]}$. 在冠醚 的主客体研究中, 联吡啶盐衍生物 $\left(N, N^{\prime}\right.$-二烷基-4,4'-联
吡啶)是其中一个重要的客体分子．基于单环冠醚对客 体联吡啶盐衍生物的识别作用，已经报道 ${ }^{[9]}$ 了许多准轮 烷、多聚准轮烷、轮烷、多聚轮烷和索烃的合成.

目前, 基于菜基块构筑的冠醚主体分子主要为菜基 的 2, 3 位 ${ }^{[10]}$, 主要研究其识别和组装性质; 而基于萗基 块 2,7 位的冠醚主体分子构筑还没有被报道. 最近我

* E-mail: zongqs@iccas.ac.cn

Received November 20, 2012; revised December 4, 2012; published online December 10, 2012.

Project supported by the China Postdoctoral Science Foundation (No. 2011M500989) and the Excellent Yong Teachers Program of Zhejiang Province (No. 00511024).

中国博士后科学基金面上基金(No. 2011M500989)、浙江省高校优秀青年教师(No. 00511024)资助项目. 
们 $^{[11]}$,报道了基于荟基块新型开链冠醚的合成及晶体结 构研究由于这个开链冠醚结构的侧链较短对联吡啶盐 没有络合作用. 因此, 我们基于富电子的 2, 7 位菜基块 设计并合成了具有更大空腔的新型冠醚二萗并[30]-冠8 (4) (Scheme 1), 并研究了其晶体结构及其对联吡啶盐 的络合性能(图 1).
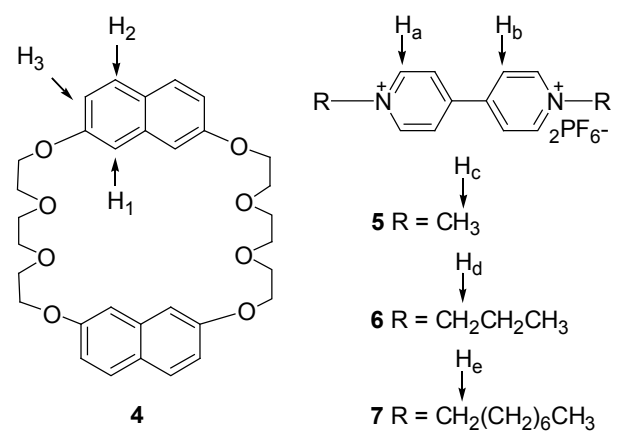

图 1 主体分子 $\mathbf{4}$ 和客体分子 $5 \sim 7$ 的结构及氢质子的归属 Figure 1 Structures and protons designations of the host $\mathbf{4}$ and guests $\mathbf{5} \sim \mathbf{7}$

\section{1 实验部分}

\section{1 试剂及仪器}

Bruker 400 型核磁共振仪[德国布鲁克公司，内标 $\mathrm{TMS}$, 溶剂为氝代氯仿 $\left(\mathrm{CDCl}_{3}\right)$ 或氝代乙腈 $\left.\left(\mathrm{CD}_{3} \mathrm{CN}\right)\right]$, SGW X-4 型熔点仪测定(温度计未校正), PE 2400 II 型 CHNS/O 元素分析仪(美国 Perkin Elmer 公司), UltimaTm Global MICROMASS-TOF 质谱仪, MERCURY CCDAFC8 单晶 X 射线衍射仪(日本). 2,7-二羟基萗(南通柏鸣 化工有限公司)、8-(对甲苯磺酰氧基)-3,6-二氧-1-辛醇(自 制, 按照文献[12]合成), 其他试剂和溶剂均为市售分析 纯或化学纯.

\section{2 化合物的合成}

1.2.1 2,7-二[8-(对甲苯磺酰氧基)-3,6-二氧- 1-辛氧 基]菜 $(3)$ 的合成

在 $100 \mathrm{~mL}$ 单口烧瓶中加入 $2.00 \mathrm{~g}$ (12.5 mmol) 2,7二羟基菜(1), $8.50 \mathrm{~g}$ (28.0 mmol) 8-对甲苯磺酰氧基-3,6-

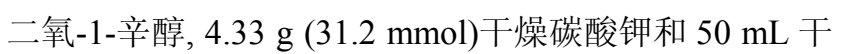

燥乙腈, 氮气保护加热回流反应 $24 \mathrm{~h}$ 至反应结束. 反应 液冷却至室温、过滤除去碳酸钾、滤液减压浓缩得 $\mathbf{6}$ 的 粗品. 不经分离, 直接将 2 的粗品溶于 $60 \mathrm{~mL}$ 干燥的二 氯甲烷进行下一步反应. 在冰浴下加入 $11.12 \mathrm{~g}(48.0$ $\mathrm{mmol})$ 氧化银、7.5 g (39.7 mmol)对甲苯磺酰氯和 $1.17 \mathrm{~g}$ (7.3 mmol)碘化钾, 保温搅拌 $1 \mathrm{~h}$ 后撤去冰浴, 混合物升 至室温继续反应 $8 \mathrm{~h}$. 反应完毕后, 在混合物中加 $10 \mathrm{~g}$ 硅胶(200 300 目)过滤、滤饼用乙酸乙酯洗涤、减压蒸 馏除去溶剂, 剩余物快速柱层析[乙酸乙酯/石油醚 $(V$ : $V=2: 1)$ ]分离得到 $7.87 \mathrm{~g}$ 油状产品 3 , 产率为 $86 \% .{ }^{1} \mathrm{H}$ NMR $\left(\mathrm{CDCl}_{3}, 400 \mathrm{MHz}\right) \delta: 2.40\left(\mathrm{~s}, 6 \mathrm{H}, \mathrm{CH}_{3}\right), 3.62(\mathrm{t}, J=$ 4.0, $5.0 \mathrm{~Hz}, 4 \mathrm{H}, \mathrm{OCH}_{2}$ ), 3.69 (q, $J=4.5,5.0 \mathrm{~Hz}, 8 \mathrm{H}$, $\left.\mathrm{OCH}_{2}\right), 3.87\left(\mathrm{t}, J=4.5,5.0 \mathrm{~Hz}, 4 \mathrm{H}, \mathrm{OCH}_{2}\right), 4.15(\mathrm{t}, J=4.5$, $\left.5.0 \mathrm{~Hz}, 4 \mathrm{H}, \mathrm{OCH}_{2}\right), 4.21\left(\mathrm{t}, J=4.5,5.0 \mathrm{~Hz}, 4 \mathrm{H}, \mathrm{OCH}_{2}\right.$ ), $7.01(\mathrm{dd}, J=2.5,8.0 \mathrm{~Hz}, 2 \mathrm{H}, \mathrm{ArH}), 7.04$ (d, $J=2.0 \mathrm{~Hz}$, 2H, ArH), 7.30 (d, $J=8.0 \mathrm{~Hz}, 4 \mathrm{H}, \operatorname{ArH}), 7.63$ (d, $J=9.0$ $\mathrm{Hz}, 2 \mathrm{H}, \mathrm{ArH}), 7.78$ (d, $J=8.5 \mathrm{~Hz}, 4 \mathrm{H}, \mathrm{ArH}) ;{ }^{13} \mathrm{C} \mathrm{NMR}$ $\left(\mathrm{CDCl}_{3}, 100 \mathrm{MHz}\right) \delta: 21.6,67.4,68.7,69.3,69.8,70.6$, $70.8,106.3,116.4,124.4,127.9,129.1,129.8,132.9$, 135.8, 144.8, 157.3; MS (ESI) $m / z: 733.24[\mathrm{M}+\mathrm{H}]^{+}$. Anal. calcd for $\mathrm{C}_{36} \mathrm{H}_{44} \mathrm{O}_{12} \mathrm{~S}_{2}$ : C 59.00, H 6.05; found C 59.04, H 6.03

\subsection{2 新型冠醚二䒬并[30]-冠-8 (4)的合成}

在 $500 \mathrm{~mL}$ 三口烧瓶中加入 $3.7 \mathrm{~g}$ 碳酸铯 $(11.5 \mathrm{mmol})$ 和 $300 \mathrm{~mL}$ 干燥 $N, N$-二甲基甲酰胺(DMF), 磁力搅拌和 氮气保护下升温至 $90{ }^{\circ} \mathrm{C}$, 再将 $3.92 \mathrm{~g}(5.4 \mathrm{mmol}) 3$ 和 $0.864 \mathrm{~g}$ (5.4 mmol) 1 溶于 $100 \mathrm{~mL}$ 干燥 DMF 的混合溶液 慢慢滴加上述溶液中, $8 \mathrm{~h}$ 滴完并继续保温反应 $72 \mathrm{~h}$. 反 应完毕后, 混合物冷却至室温、过滤除去碳酸铯、减压 蒸馏除去溶剂, 剩余物快速柱层析[二氯甲烷/乙酸乙酯 $(V: V=20: 1)$ ]分离得到 $1.32 \mathrm{~g}$ 白色固体 4 , 产率 $42 \%$. m.p. $258.0 \sim 260.0{ }^{\circ} \mathrm{C} ;{ }^{1} \mathrm{H}$ NMR $\left(\mathrm{CDCl}_{3}, 400 \mathrm{MHz}\right) \delta$ : $3.762\left(\mathrm{~s}, 8 \mathrm{H}, \mathrm{OCH}_{2}\right), 3.88\left(\mathrm{t}, J=4.8,4.4 \mathrm{~Hz}, 8 \mathrm{H}, \mathrm{OCH}_{2}\right)$, $4.10\left(\mathrm{t}, J=4.4,5.2 \mathrm{~Hz}, 8 \mathrm{H}, \mathrm{OCH}_{2}\right), 6.89$ (d, $J=2.0 \mathrm{~Hz}$, $4 \mathrm{H}, \mathrm{ArH}), 7.00$ (dd, $J=2.4,6.4 \mathrm{~Hz}, 4 \mathrm{H}, \mathrm{ArH}), 7.57$ (d, $J=$ $8.8 \mathrm{~Hz}, 4 \mathrm{H}, \mathrm{ArH}) ;{ }^{13} \mathrm{C} \mathrm{NMR}\left(\mathrm{CDCl}_{3}, 100 \mathrm{MHz}\right) \delta: 67.34$,

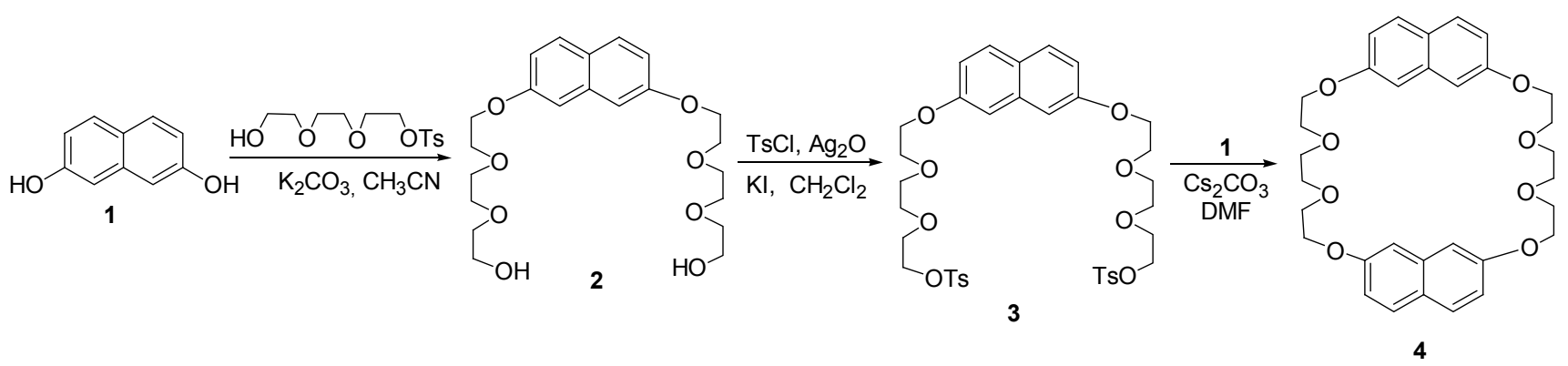

Scheme 1 
$69.68,71.14,106.43,116.35,124.37,129.04,135.77$, 157.29; MS (ESI) $m / z: 549.25[\mathrm{M}+\mathrm{H}]^{+}$. Anal. calcd for $\mathrm{C}_{32} \mathrm{H}_{36} \mathrm{O}_{8}$ : C 70.06, H 6.61; found C 70.10, H, 6.59.

\section{3 化合物 4 的晶体培养和结构测定}

将化合物 4 溶解在氯仿和乙腈(体积比为 $2: 1$ )中配 成适当的稀溶液置于三角雉形瓶中, 用滤纸覆盖瓶口, 室温静置待溶剂自然挥发得所需晶体. 取 $0.50 \mathrm{~mm} \times$ $0.50 \mathrm{~mm} \times 0.40 \mathrm{~mm}$ 的无色晶体置于石墨单色化 Mo K $\alpha$ $(\lambda=0.071070 \mathrm{~nm})$ 辐射为光源, 在 $293(2) \mathrm{K}$ 下以 $\omega / 2 \theta$ 方 式扫描, 在 $3.0^{\circ} \leqslant \theta \leqslant 25.3^{\circ},-9 \leqslant h \leqslant 9,-16 \leqslant k \leqslant 16$, $-15 \leqslant l \leqslant 16$ 的范围内收集到 12870 个衍射数据, 其中 4920 个为独立数据 $\left(R_{\mathrm{int}}=0.026\right)$, 可观察衍射点 3755 个 $[I>2 \sigma(I)]$, 晶体结构由直接法解出, 对全部非氢原子的 坐标及各向异性参数用 SHELXS- $97^{[13,14]}$ 程序以最小二 乘法修正 $F^{2}$ 进行精修, 最终偏离因子 $R=0.053, w R=$ 0.126. 化合物 4 在剑桥晶体结构数据库的编号为 CCDC910861.

\section{2 结果与讨论}

\section{1 合成}

Scheme 1 所示是目标分子 4 的合成路线. 以 2,7 二 羟基萗为起始原料, 在碳酸钾作碱下先与 8 -(对甲苯磺 酰氧基)-3,6-二氧-1-辛醇进行亲核取代反应得 2 粗品, 2 粗品无需分离直接与对甲苯磺酰氯反应得 $\mathbf{3}$, 该反应中 用到氧化银和碘化钾, 其中氧化银做碱、碘化钾为催化 剂. 3 最后与 2,7-二差基萗进行成环反应得到目标主体 分子 4, 三步总收率为 $35.0 \%$. 产物分别经 ${ }^{1} \mathrm{H} \mathrm{NMR},{ }^{13} \mathrm{C}$ NMR, 元素分析, 质谱和 X 射线衍射的证实. 制备 7 的 后处理过滤需要加入少量硅胶, 主要目的是使过滤比较 快速, 如果不加硅胶滤渣比较粘稠从而导致过滤困难. 最后一步成环反应的浓度保持在 $10 \sim 20 \mathrm{mmol} / \mathrm{L}$ 之间反 应收率最高, 且需要氮气保护. 反应中所需要的溶剂都 需要经过 $4 \AA$ 分子篮干燥处理.

\section{2 晶体结构}

化合物 4 的晶体分子式为 $\mathrm{C}_{32} \mathrm{H}_{3} \mathrm{O}_{8}$, 分子量为 548.61. 单晶 $\mathrm{X}$ 射线衍射测试表明该晶体属三斜晶系 (Triclinic), $P-1$ 空间群, 晶胞参数分别为 $a=8.165(3) \AA$, $b=13.435(4) \AA, c=14.083(3) \AA, \alpha=64.11(2)^{\circ}, \beta=$ $80.40(3)^{\circ}, \gamma=88.19(3)^{\circ}, V=1368.9(7) \AA^{3}, Z=2, D_{\mathrm{c}}=$ $1.331 \mathrm{~g} / \mathrm{cm}^{3}, \lambda=0.071070 \mathrm{~nm}, \mu($ Mo $\mathrm{K} \alpha)=1.331 \mathrm{~mm}^{-1}$, $M_{\mathrm{r}}=353.22, F(000)=584$. 化合物的晶体结构见图 2, 晶 胞堆积图见图 4.

在晶体结构中, 2 个荎环的 2,7 位分别通过 2 个二缩 三乙二醇醚键相连形成 1 个二荎并[30]-冠-8 的大环结

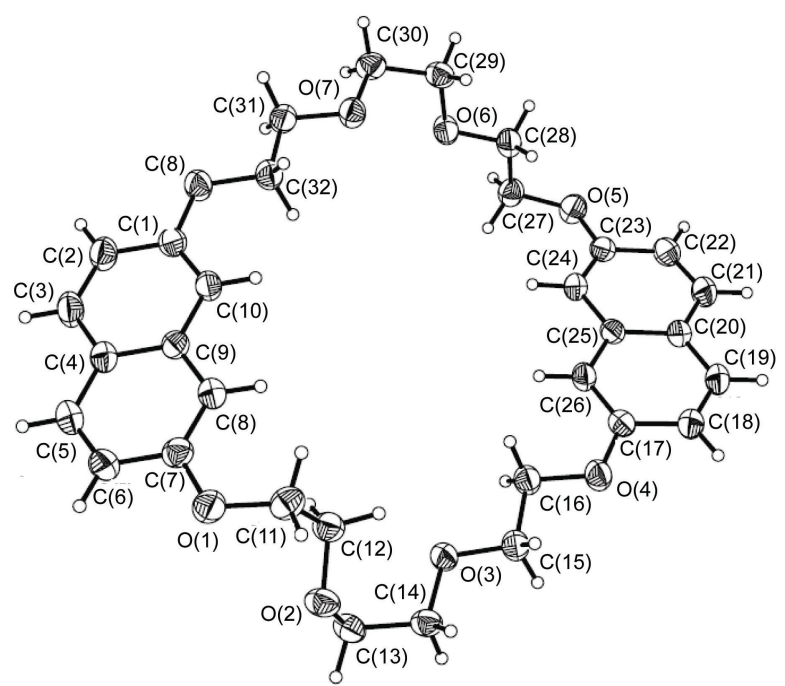

图 2 主体分子 $\mathbf{4}$ 的晶体结构图,

Figure 2 Crystal structure of 4

构, 环的空腔大小为 $8.2 \AA \times 12.0 \AA$, 分子呈 $\mathrm{Z}$ 型结构. 两个萗环 $[C(1)-C(10)$ 和 $C(17)-C(26)]$ 之间二面角为 16.5(2) . 分子间通过 $\mathrm{C}-\mathrm{H} \cdots \mathrm{O}$ 氢键作形成二聚体(图 3 和表 1), 有趣的是这些二聚体通过进一步的 $\mathrm{C}-\mathrm{H} \cdots \pi$ 相互作用叠加成二维层状结构平行于 $a b$ 平面(图 4).

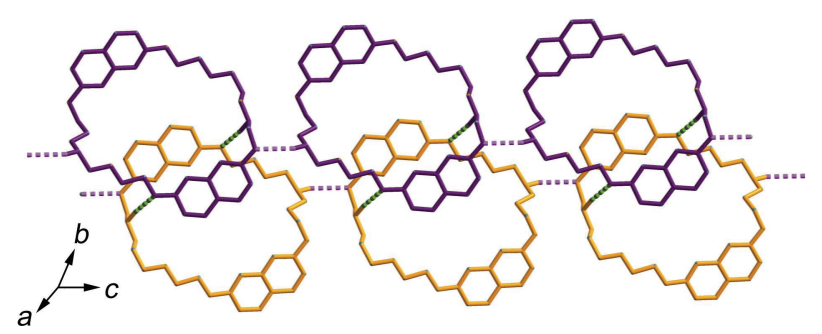

图 3 化合物 4 的氢键环状二聚体

Figure 3 Cyclic hydrogen-bonded dimer in the title compound 4

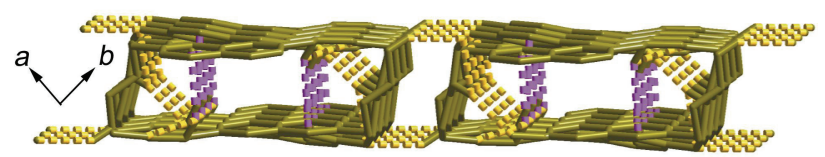

图 4 二聚体叠加成的二维层状结构

Figure 4 Two-dimensional sheets parallel aggregated by dimers

表 1 氢键键长 $(\AA)$

Table 1 Hydrogen-bond geometry

\begin{tabular}{lcccc}
\hline \multicolumn{1}{c}{$D-\mathrm{H} \cdots A$} & $D-\mathrm{H}$ & $\mathrm{H} \cdots A$ & $D \cdots A$ & $D-\mathrm{H} \cdots A$ \\
\hline $\mathrm{C}(13)-\mathrm{H}(13 \mathrm{~B}) \cdots \mathrm{O}(5)^{a}$ & 0.97 & 2.57 & $3.432(3)$ & 148 \\
$\mathrm{C}(28)-\mathrm{H}\left(28^{a}\right) \cdots(\mathrm{O} 4)^{b}$ & 0.97 & 2.48 & $3.425(3)$ & 165 \\
$\mathrm{C}(16)-\mathrm{H}(16 \mathrm{~B}) \cdots \mathrm{C}(\mathrm{g} 4)^{b}$ & 0.97 & 2.88 & $3.704(2)$ & 144 \\
$\mathrm{C}(32)-\mathrm{H}\left(32^{a}\right) \cdots \mathrm{C}(\mathrm{g} 2)^{c}$ & 0.97 & 2.82 & $3.592(4)$ & 138 \\
\hline${ }^{a} x-1, y, 1+\mathrm{z} ;{ }^{b} 2-x, 2-y,-z ;{ }^{c} 2-x, 2-y, 1-z$.
\end{tabular}




\section{3 识别性能初步研究}

通过核磁滴定分别研究了主体分子 $\mathbf{4}$ 对缺电子联吡 啶盐 $5 \sim 7$ (烷基链长度越来越长的顺序)在溶液中的相互 络合作用, 溶剂为混合溶剂[氞代氯仿/氞代乙腈 $(V$ : $V=2: 1]$, 温度为室温. 当向主体 4 的溶液中加入 1 equiv. 的客体分子 $\mathbf{5}$ 时, 溶液的颜色立即变成橙色, 主要 是因为富电子的主体 $\mathbf{4}$ 和缺电子的甲基联吡啶盐 $\mathbf{5}$ 之间 发生了电荷转移. 当等物质的量的主体 4 和联吡啶盐 6 或 7 混合时溶液的颜色也变成橙色，同样说明了主客体 之间发生了电荷转移; 而主体与 3 个不同客体分子络合 的颜色变化基本相同, 则说明主体分子对 3 个不同客体 分子的识别作用相当. 通过向主体中 4 不断加入客体的 核磁滴定实验, 主要观察主体上氢质子的化学位移变

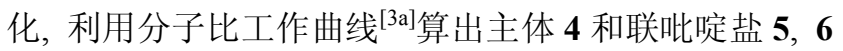
和 7 的分子作用比都是 $1: 1$.

如图 5 所示, 当向主体 $\mathbf{4}$ 中加入 1 equiv. 的 $\mathbf{5}$ 时, 氢 谱发生了非常明显的变化(图 5b): 主体上 $\mathrm{H}_{1}, \mathrm{H}_{2}, \mathrm{H}_{3}$ 和 客体上的 $\mathrm{H}_{\mathrm{a}}$ 及 $\mathrm{H}_{\mathrm{b}}$ 明显向高场发生了移动, 化学位移变 化值见表 2; 而客体 $N$-甲基上的 $\mathrm{H}_{\mathrm{c}}$ 基本无变化. $\mathrm{H}_{1}, \mathrm{H}_{2}$, $\mathrm{H}_{3}, \mathrm{H}_{\mathrm{a}}$ 和 $\mathrm{H}_{\mathrm{b}}$ 向高场移动了比较大的化学位移说明了主 客体芳环之间存在比较强的 $\pi-\pi$ 相互作用, 而 $\mathrm{H}_{\mathrm{c}}$ 基本无 变化说明甲基上的氢原子没有与主体分子上的冠醚氧 原子形成氢键. 从而可以推断在溶液中联吡啶盐是从主 体分子 $Z$ 形空穴中穿插而过. 同样当向主体 $\mathbf{4}$ 的混合溶 剂中加入一当量的 6 或 7 时, 主体上 $\mathrm{H}_{1}, \mathrm{H}_{2}, \mathrm{H}_{3}$ 和客体上 的 $\mathrm{H}_{\mathrm{a}}$ 及 $\mathrm{H}_{\mathrm{b}}$ 明显向高场发生了移动. 由表 2 可以看出, 主 体分子与不同联吡啶盐化学位移变化值基本相同, 也说 明了络合性能相似. 另外, 我们利用 Scatchard 曲线 ${ }^{[15]}$ 计算出它们的络合常数 $\left(K_{\mathrm{a} 4} 4_{5}, K_{\mathrm{a}} 4^{+6}\right.$ 和 $\left.K_{\mathrm{a} 44^{+7}}\right)$ 分别为 $(3.53 \pm 0.2) \times 10^{2},(3.26 \pm 0.3) \times 10^{2}$ 和 $(3.15 \pm 0.2) \times 10^{2}$ $\mathrm{L} / \mathrm{mol}$, 络合常数变化不大, 进一步证明了主体分子 $\mathbf{4}$ 对

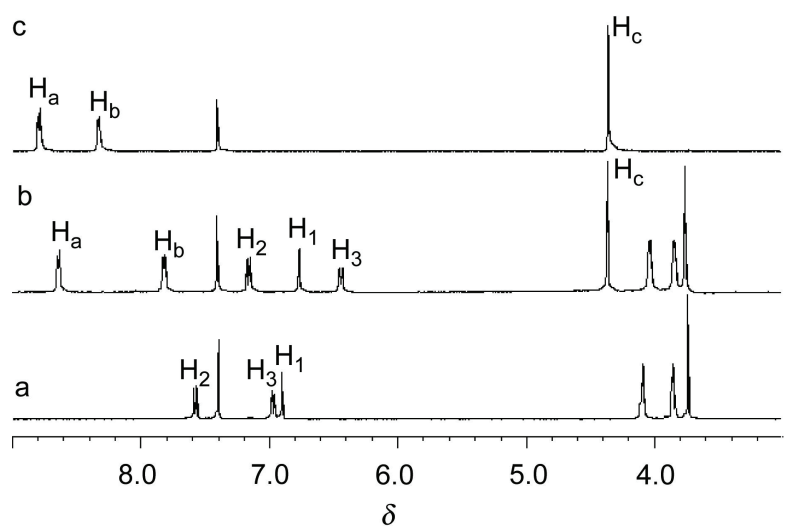

图 5 室温下在 $\mathrm{CD}_{3} \mathrm{CN} / \mathrm{CDCl}_{3}(V: V=1: 2)$ 中的部分氢谱图 $(400 \mathrm{NHz})$

Figure 5 Partial ${ }^{1} \mathrm{H}$ NMR spectra $(400 \mathrm{MHz})$ in $\mathrm{CD}_{3} \mathrm{CN}$ / $\mathrm{CDCl}_{3}(V: V=1: 2)$

(a) Free host 1, (b) 4 and 1.0 equiv. of 5, (c) free guest 5. [4 $]_{0}=8 \mathrm{mmol} / \mathrm{L}$
客体分子联吡啶盐不具有选择性识别作用.

表 2 化学位移变化

Table 2 Change of chemical shift

\begin{tabular}{cccccc}
\hline Host + Guest & $\Delta \delta_{\mathrm{H}_{1}}$ & $\Delta \delta_{\mathrm{H}_{2}}$ & $\Delta \delta_{\mathrm{H}_{3}}$ & $\Delta \delta_{\mathrm{H}_{\mathrm{a}}}$ & $\Delta \delta_{\mathrm{Hb}_{\mathrm{b}}}$ \\
\hline $\mathbf{4 + 5}$ & 0.15 & 0.44 & 0.58 & 0.21 & 0.52 \\
$\mathbf{4}+\mathbf{6}$ & 0.13 & 0.44 & 0.56 & 0.20 & 0.51 \\
$\mathbf{4}+\mathbf{7}$ & 0.11 & 0.42 & 0.54 & 0.20 & 0.50 \\
\hline
\end{tabular}

\section{3 结论}

本文报道了基于荎基块新型二荎并[30]-冠-8 冠醚 主体分子 4 合成，产物通过 ${ }^{1} \mathrm{H} N M R,{ }^{13} \mathrm{C} N M R$, 元素分 析, 质谱和 X 射线衍射进行了结构表征, 并初步研究了 其对联吡啶盐识别性能. 目前, 该主体分子对其它客体 分子识别性能及组装工作正在研究当中.

\section{References}

[1] (a) Pedersen, C. J. J. Am. Chem. Soc. 1967, 89, 2495. (b) Pedersen, C. J. J. Am. Chem. Soc. 1967, 89, 7017.

[2] Liu, Y.; You, C. C.; Zhang, H. Y. Supramolecular Chemistry, Nankai University Publication, Tianjin, 2001 (in Chinese). (刘育, 尤长城, 张衡益, 超分子化学, 南开大学出版社, 天津, 2001.)

[3] (a) Zong, Q. S.; Chen, C. F. Org. Lett. 2006, 8, 211.

(b) Zong, Q. S.; Zhang, C.; Chen, C. F. Org. Lett. 2006, 8, 1859.

(c) Han, Y.; Lu, H. Y.; Zong, Q. S.; Guo, J. B.; Chen, C. F. J. Org. Chem. 2012, 77, 2422.

(d) Zhao, J. M.; Zong, Q. S.; Chen, C. F. J. Org. Chem. 2010, 75, 5092 .

(e) Su, Y. S.; Chen, C. F. Org. Lett. 2010, 12, 1888.

[4] Tang, C. C.; Wang, L. H.; Yun, Y. B.; Zhang, C. L.; Liu, B. Q. Acta Chim. Sinica 2011, 69, 343 (in Chinese).

(唐橙橙, 王丽华, 贠延滨, 张陈淋, 刘必前, 化学学报, 2011, 69, 343.)

[5] Zhang, Q.; Xu, J. Acta Chim. Sinica 2011, 69, 2287 (in Chinese). (张强, 许娟, 化学学报, 2011, 69, 2287.)

[6] Ye, Q. J.; Yang, F. F.; Zhang, Y. Y.; Jiao, Z. Y.; Guo, H. Y. Chin. J. Org. Chem. 2012, 32, 189 (in Chinese).

(叶进启, 杨发福, 张䈶逸, 焦自玉, 郭红玉, 有机化学, 2012, 32,189 .)

[7] (a) Huang, F. H.; Zakharov, L. N.; Rheingold, A. L.; Ashraf-Khorassani, M.; Gibson, H. W. J. Org. Chem. 2005, 70, 809.

(b) Huang, F. H.; Gibson, H. W.; Bryant, W. S.; Nagvekar, D. S.; Fronczek, F. R. J. Am. Chem. Soc. 2003, 125, 9367.

(c) Pederson, A. M. P.; Ward, E.; Schoonover, D. S.; Slebodnick, C.; Gibson, H. W. J. Org. Chem. 2008, 73, 9094.

(d) Zhang, M. M.; Zhu, K. L.; Huang, F. H. Chem. Commun. 2010, 46, 8131 .

(e) Zhang, M. M.; Zheng, B.; Huang, F. H. Chem. Commun. 2011, 47, 10103.

[8] (a) Chen, L.; Zhang, Y. M.; Liu, Y. J. Phys. Chem. B 2012, 116, 9500 .

(b) Chen, L.; Zhang H. Y.; Liu Y. J. Org. Chem. 2012, 77, 9766.

[9] (a) Sauvage, J. P.; Dietrich-Buchecker, C. Molecular Catenanes, Eds.: Rotaxanes and Knots, Wiley-VCH, Weinheim, Germany, 1999.

(b) Amabilino, D. B.; Stoddart, J. F. Chem. Rev. 1995, 95, 2725. 
(c) Balzani, V.; Credi, A.; Raymo, F. M.; Stoddart, J. F. Angew. Chem., Int. Ed. 2000, 39, 3348.

[10] (a) Suresh, M.; Mandal, A. K.; Kesharwani, M. K.; Adarsh, N. N.; Ganguly, B.; Kanaparthi, R. K.; Samanta, A.; Das, A. J. Org. Chem. 2011, 76, 138.

(b) Mandal, A. K.; Das, P.; Mahato, P.; Acharya, S.; Das, A. J. Org. Chem. 2012, 77, 6789 .

(c) Davidson, G. J. E.; Sharma, S.; Loeb, S. J. Angew. Chem., Int. Ed. 2010, 49, 4938.

(d) Hubbard, A. L.; Davidson, G. J. E.; Patel, R. H.; Wisner, J. A.; Loeb, S. J. Chem. Commun. 2004, 39, 138.
[11] Zong, Q. S.; Wu, J. Y. Chin. J. Org. Chem. 2012, 32, 1509 (in Chinese).

(宗乾收, 吴建一, 有机化学, 2012, 32, 1509.)

[12] Boerjesson, L.; Welch, C. J. Acta Chem. Scand. 1991, 45, 621.

[13] Sheldrick, G. M. SHELXS97, A Program for Automatic Solution of Crystal Structure, University of Göttingen, Germany, 1997.

[14] Sheldrick, G. M. SHELXS97, A Program for Crystal Structure Refinement, University of Göttingen, Germany, 1997.

[15] Connors, K. A. Binding Constants, J. Wiley and Sons, New York, 1987.

(Qin, X.) 